\title{
Human Impacts and Management of Carbon Sources
}

\section{Executive Summary}

The energy system dominates human-induced carbon flows on our planet. Globally, six billion tons of carbon are contained in the fossil fuels removed from below the ground every year. More than $90 \%$ of the carbon in fossil fuels is used for energy purposes, with carbon dioxide as the carbon product and the atmosphere as the initial destination for the carbon dioxide. Significantiy affecting the carbon flows associated with fossil fuels is an immense undertaking.

Four principal technological approaches are available to affect these carbon flows:

- Fossil fuels and other energy resources can be utilized more efficiently.

- Energy sources other than fossil fuels can be used.

- Carbon dioxide from the combustion of fossil fuels can be trapped and redirected, preventing it from reaching the atmosphere (fossil carbon sequestration).

- One can work outside the energy system to remove carbon dioxide biologically from the atmosphere (biological carbon sequestration).

An optimum carbon management strategy will surely implement all four approaches and a wise R\&D program will have vigorous sub-programs in all four areas. These programs can be effective by integrating scenario analyses into the planning process. A number of future scenarios must be evaluated to determine the need for the new technologies in a future energy mix. This planning activity must be an iterative process.

At present, $R \& D$ in the first two areas-energy efficiency and non-fossil fuel energy resources-is relatively well developed. By contrast, $R \& D$ in the third and the fourth areas-the two carbon sequestration options-is less well developed. The task before the workshop was to recommend ways to initiate a vigorous carbon sequestration research program without compromising the strength of the current programs in the first two areas.

We recommend that this task be fulfilled by initiating several new programs in parallel. First, we recommend that a vigorous carbon sequestration program be launched. We have confidence that the time is ripe for this new undertaking. Several studies conducted over the past two years have scoped out the research issues that need to be explored and have revealed a wide variety of technological approaches that call out for detailed analysis and field testing.

Second, we recommend that $R \& D$ efforts in the areas of efficient energy use and clean energy (technologies not using fossil resources or significantly reducing carbon emissions per unit of energy generated) be maintained and strengthened. The lead times necessary for market penetration of successful technologies when they are needed require a robust federally funded $R \& D$ program.

Third, we recommend that a broad carbon management research program be properly integrated into all four of the approaches listed above. Specifically, we recommend four elements of such a program:

- A program in support of decision-oriented research, emphasizing life-cycle analysis systems and risk analysis, with the concomitant development of tools for technology assessment, cross-technology comparison, and analysis of externalities.

- A program designed to support a small number of research centers, each focusing on a specific area of carbon management, creatively combining several disciplinary approaches and featuring strong industry participation.

- A program in support of investigator-initiated research.

- A program focused on effective means of engaging the public.

All of these initiatives must give considerable weight to the consideration of the social implications of the technologies under investigation. We believe that public acceptance will be and should be a critical determinant of the evolution of the technologies, whose promise the proposed program is designed to explore.

Human Impacts and Management of Carbon Sources-15 


\section{Tindings}

The participants in the workshop agreed that there was a need for a long-term view for R\&D. Given world oil, coal, and gas prices and reserves, it is apparent that we are entering another "Fossil Fuel Century." It is also apparent that, in order to develop new technologies for carbon sequestration, the lead time for their insertion into complementary energy conversion technologies must be measured in decades.

Many technologies can be economically competitive in the near term. These are primarily in the areas of energy-efficient technologies (which reduce energy use per gross domestic product) and decarbonization or clean fuel technologies (which reduce $\mathrm{CO}_{2}$ emissions per Btu). Some sequestration technologies, such as reforestation, are being implemented today. Others may only be implementable after 2025 .

For most of these technologies, it is too early to pick winners and losers. Some technologies may be economically viable in the near term. Other, truly innovative technologies may achieve significant breakthroughs with the ability to capture, separate, and sequester significant amounts of $\mathrm{CO}_{2}$ for reasonable costs. Economic viability must sometimes be balanced by energy output. For example, decarbonizing fossil fuels prior to combustion may result in economically viable byproducts, while doing so following combustion may result in greater energy output. All of these technologies must also be environmentaliy benign, socially acceptable, and operationally efficient.

A broad-based approach to fundamental and supporting scientific research and technology development is the best path forward because we have time to "do this right."

At this time, sequestration technologies are the newest set of tools in the tool kit to mitigate $\mathrm{CO}_{2}$ emissions. Sequestration currently suffers from high costs, lack of public acceptance, and uncertain risks associated with geological and ocean disposal. Energyefficient and decarbonization technologies have been developed with other drivers in mind, such as mitigation of other environmental emissions, national security concerns, and cost concerns. Sequestration is affected by none of these. Sequestration technology will allow for continued use of fossil fuels. R\&D activities for sequestration must also be accompanied by supporting research. These areas include environmental impacts, ecosystem dynamics, public health and safety, epidemiology, and toxicology. All sequestration, capture, and separations technologies will need to incorporate new methods of monitoring to evaluate the effectiveness of the technology, as well as new simulation, engineering, and fate and transport models for predicting the effectiveness of the technology.

There was broad agreement from participants of the workshop that sequestration technologies would not enter the marketplace without effectively engaging the public as to their current and future need. This is analogous to other public technological issues such as those related to nuclear energy. Based on eventual public acceptance, it was believed that several carbon management pathways will result because of the need for market acceptance and various related incentives such as tax reduction, emissions regulations, and economic incentives. More advanced sequestration technologies should result in viable products that will result in collateral economic benefits.

\section{Recommendations}

\section{Initiate an $R \& D$ Program in Carbon Sequestration}

Carbon sequestration is an important technology option for allowing fossil fuels to maintain a substantial share of the future energy market. Our recommendation is the creation of a program to develop technically feasible sequestration options for meeting economic, environmental, and security needs. The scope of the research should range from fundamental research to technology development and testing as well as demonstrations of pilot-plant implementations. Because the field is still very new, it would be premature to pick winners among the various approaches that have been suggested. Major research issues that have been identified are:

- Capture and separation of $\mathrm{CO}_{2}-$ Capture and separation are preconditions to many sequestration options. Adsorption, absorption, and membrane technologies are examples. Fuel decarbonization leading to hydrogen and either carbon or carbon dioxide represents a separation technology that would naturally lead to a hydrogen-based economy.

- Sequestration in soils and vegetation-Enhanced uptake by soils and vegetation could offset carbon dioxide emissions on a relatively short time scale.

- Storage in geological formations-Storage in geological reservoirs can sequester $\mathrm{CO}_{2}$ over rather 
long periods of time but raises issues that include storage times, migration and transport, effects of temperature and $\mathrm{pH}$, and the chemical reactions with the media, which may be desirable or harmful.

- Ocean disposal-Ocean disposal represents an option for very long-term sequestration of $\mathrm{CO}_{2}$. However, there are similar concerns about the benefits and risks related to this approach as there are about geological sequestration.

- Advanced chemical methods to transform carbon dioxide to stable forms-Advanced chemical methods allow for sequestration of other forms of carbon, ranging from elemental carbon over organic durable compounds like plastics to inorganic mineral carbonates that form exothermically from carbon dioxide.

- Advanced biological methods of sequestrationPhotosynthesis is one of the largest sinks of atmospheric $\mathrm{CO}_{2}$ and would allow sequestration from dispersed sources. Enhancement of this sink by innovative biological approaches, possibly using concentrated streams of $\mathrm{CO}_{2}$, could provide a substantial carbon sequestration potential.

All approaches need to address safety, environmental impacts, effectiveness over time, legacies for future generations, capacity, economic viability, and social acceptance. Economic viability can be enhanced by useful byproducts, although the scale of the sequestration process makes it likely that the majority of sequestration will produce products of presently low value.

Enhance Research, Development, and Technology in Energy Efficiency and Clean Energy

Although sequestration is a relatively new approach, government and industry have supported R\&D in enduse energy efficiency and clean energy but not necessarily with carbon management as the driver. Reduced oil imports, improved urban and regional air quality, reduced costs of energy generation and energy end-use have been and continue to be national needs and thus drivers for the R\&D.

Our recommendation is to enhance these programs to meet a goal of $50 \%$ carbon reductions through the use of these technologies by 2050 .

In the case of energy end-use, carbon emissions are divided roughly evenly among the buildings, industry, and transportation sectors. For buildings, enhanced research will lead to improved and more energy-efficient equipment and appliances, as well as building envelopes. Modern information, communication, and visualization systems can be utilized in the design, construction, and operation of buildings to reduce energy use while maintaining or improving human health, safety, and productivity.

For industry, enhanced R\&D will lead to improvements in energy conversion and utilization for industrial processes (e.g., advanced turbines), the recovery and utilization of resources (e.g., waste streams from one process as input to another), and more efficient processes themselves (e.g., improved separation processes). There are also certain enabling technologies, such as advanced materials and new chemical processes, that should be considered.

In transportation, lightweight vehicles are a main target for increased fuel economy. Incremental advances in lightweight materials, improved engines, and streamlining, for example, will lead to advanced conventional vehicles. Enhanced research will accelerate the introduction of new generations of battery-powered, hybrid, and fuel-cell vehicles. Alternative fuels (e.g., from biomass) can significantly reduce carbon emissions. Freight vehicles could have improved fuel economy through ultra-high-efficiency diesel engines and hybrid systems with the use of regenerative braking in delivery vehicles.

Clean energy technologies include renewables, nuclear, and improved processes for fossil fuels. Renewables offer a host of zero- or low-carbon options, the introduction of which will be accelerated through enhanced R\&D. These options include photovoltaics, solar thermal, wind, geothermal, advanced hydropower, biomass for electrical energy and transportation fuels, and for the longer term, advanced photoconversion processes such as for the production of hydrogen.

Advanced nuclear processes include methods for extending the life of existing nuclear power plants and developing a new generation of fission power plants that have higher efficiency, as well as addressing other issues, such as waste generation and proliferation, and for the longer term, fusion reactors.

In the case of fossil energy, research will lead to advances in fossil resource development, such as more energy-efficient oil refining, efficient processes for converting natural gas to liquids, increased natural gas production as a "low-carbon" fuel, and the use of carbon dioxide for improved oil and gas recovery. In the case of fossil fuel conversion to electricity, enhanced research 
will lead to high-efficiency coal-based power generation technologies, high-efficiency power generation (e.g., gas turbines with combined cycle), and over the longer term, "energyplexes" with zero carbon emissions.

\section{Development of an Integrated Multidisciplinary Research and Development Program}

We recommend the development of an integrated $R \& D$ program to understand how carbon management technologies could be developed and deployed over the course of the next century in a highly competitive global energy system. The goal of this research is the development of a framework that will encompass all aspects of carbon management including carbon intensity reduction, energy efficiency, and carbon sequestration. It will integrate technological, social, and ecological aspects of carbon management. This approach will improve our understanding of the potential for technology utilization, as well as critical technology pathways and barriers to their deployment.

This program includes the following research areas:

- Life-cycle analysis: To adequately understand the implications of developing and deploying new technologies on a global scale, it is essential to consider their complete life cycle. This research includes investigations of the costs, performance, and consequences of individual and supporting technologies over the entire timeframe in which the technology might be deployed. Important considerations include, for example, infrastructure, resource, and externality costs.

- Risk assessment: A complete understanding of risk is essential as we contemplate deployment of technologies that do not yet exist or are trivial constituents of the global energy system. Risks to be assessed include energy security, environment and human health risks, and operational safety of carbon management technologies. Risk assessment should take into account a complete life-cycle analysis. Risk assessment is an iterative process that acts as a continuous guide.

- Systems analysis: Energy technologies are not deployed in isolation from competing and complementing technologies. This avenue of research will help identify the potential contributions of technologies in concert with and/or in isolation from competing and complementing technologies. Systems analysis must include a broad suite of greenhouse- related emissions and interactions among other environmental consequences, energy, land-use, economy, and society.

- Technology characterization: Systems and risk analysis require usable technology characterizations. Critical technology characteristics include performance efficiency, capital intensity, operating characteristics, post-operational requirements, and potential for disrupting events. In addition, technologies must be evaluated over the full range of their possible operating characteristics. This activity will identify competitive opportunities, critical resource limitations, and barriers to market penetration and public acceptance.

- Uncertainty: Research should take uncertainty explicitly into account. There are several ways uncertainty enters, including uncertainty in the technology characterization and in technology characterizations of complementing and competing technologies, and in the large-scale energy-economysociety background in which these technologies are deployed. Effective uncertainty analysis must incorporate scenario development and analysis that evaluates the range of futures in which new technologies are proposed to be deployed.

- Ecosystem interactions: This research will examine cumulative environmental impacts from the deployment of individual and multiple technologies by incorporating research advances in toxicology, epidemiology, and the fate and transport of contaminants.

Create Cross-Disciplinary Institutes for Addressing Specific Carbon Management Issues in a

\section{Comprehensive Way}

We recommend Institutes for Clean Energy (ICE) be created to serve as a focus to properly evaluate the interrelationships between technology and related impacts on the environment and society. The following are the points supporting this recommendation.

- Justification for Institutes: The institutes will serve as a focus to integrate cross-disciplinary activities associated with this work. These will also serve as a mechanism for aggregating needed expertise and as a mechanism for allowing government, academic, and industrial experts to work together more effectively, while leveraging their collective resources and expertise. The creation of institutes allows for a focus

\footnotetext{
on pro specif

- Reque

natior

institi

havin

educa

comp

to the

impa

techr:

self-1

wou]

arise

enco

appr

theit

- Expe

inco

shor

scie

shor

gen

pro

mu

of

acc

im]

iss

on

ser

Fount

Resec

It

term

reso?

in ba

avail

and

I

the !

fede

scie

geo

scie

rect

bro

res:
} 
on problem-solutions rather than on disciplinespecific research.

- Requests for proposals would be open to universities, national laboratories, industry, and other appropriate institutions. An institute would be most successful by having a mix of these entities but must incorporate an educational component. As part of this educational component, the institute would engage and reach out to the public, while addressing socioeconomic impacts and risk perceptions associated with new technologies and processes. An institute would be self-managed, with an external advisory board, and would collaborate with other institutes as the need arises. International collaboration would also be encouraged. Institutes would be required to make appropriate use of resources, such as user facilities, of their member organizations.

- Expected Outcomes: A systems approach must be incorporated for finding solutions. This approach should accelerate the generation of new knowledge, science, simulation models, and technologies. It should serve as a platform to educate future generations of scientists and engineers and to promote activities in multi-organization and multidisciplinary settings. In this manner, the nature of collaborative cross-disciplinary research can be accelerated. All of this should be done in concert with improving public awareness of carbon management issues and encouraging improved science education on all levels, but particularly at the primary and secondary educational levels.

\section{Foundational Support for Carbon Management Research}

It is our recommendation that because of the longterm nature of the carbon management problem and its resolution, it is essential to maintain steady investments in basic research so that the scientific underpinnings are available as new technologies are developed, piloted, and implemented.

The workshop applauds the commitment made over the long haul by the NSF and DOE as key stewards of federal investment in materials science, engineering science, chemical science, biological science, geoscience, computer/information science, nuclear science, and environmental and ecological science. We recommend continued and enhanced investment in a broad portfolio of principal investigator-initiated science research-both curiosity-driven and directed.
Scientific excellence, peer review, and internationally leveraged collaborations are key criteria that we wish to endorse so that the United States will remain on the scientific leading edge in fields essential to our national success and well-being. We recognize that as carbon management technologies evolve over time, the portfolio of directed basic research must continue to evolve to ensure relevance, focus, and value.

A reiteration of basic research needs, both now and in the future, is not included in this report because a number of high-quality, focused studies and strategic planning exercises have been conducted within the scientific community and agency programs. We endorse such a broad-based inclusive process and their recommendations. We ask that carbon management technologies move to the forefront as one of the country's key drivers for future basic research investments.

\section{Engaging the Public}

Our group recommends that in order to achieve success with the carbon management problem we will need to have public acceptance. To achieve this, one must go well beyond educating the public: we need to engage the public in a full discussion of all the issues associated with the carbon problem. The Knowledge Dissemination and Knowledge Networks group is already addressing the details of how this can be accomplished. We would like to stress a few key points that must be considered as part of this process.

The energy industry is currently extremely large and is expected to grow by a large factor in the next 50 to 100 years as the world population doubles and as the rest of the world, which dominates the global population, attempts to catch up with the standard of living of the more developed nations. The energy industry has an enormous impact on the environment, and this is likely to grow in the future. Any change in the way the industry operates will bring with it new environmental impacts that will be closely scrutinized as they differ from the status quo. The general public must be included in the discussion of all the issues associated with the $\mathrm{CO}_{2}$ problem and any proposed solutions. Given today's climate of concern about the environment and general public skepticism of technological fixes, engaging the public and gaining its blessing for any solution will be critical to that solution's acceptance. The sooner we begin this process, the more trust we will be able build, and the greater the chance that we will be

Human Impacts and Management of Carbon Sources-19 
able to find solutions that will both be effective and have a chance of being adopted.

A number of topics will form the cornerstone of the needed public engagement. Among these are a clear perspective about the nature of the $\mathrm{CO}_{2}$ probiem, whose effects grow slowly and will not necessarily be generally noticed on a daily basis. The problem is further hidden by the scale of natural fluctuations. It is especially important to communicate the scale of the problem and how it will grow in the future. Finally, the time scale for changing something as large as the overall energy industry naturally sets a substantive time scale for a solution to become effective. 